\title{
Génétique de l'asthme
}

L'asthme est une maladie multifactorielle dépendant à la fois de facteurs génétiques et d'éléments liés à l'environnement. Il s'agit d'une infection étroitement associée à l'hyperréactivité bronchique et à l'allergie, deux anomalies comportant également une composante génétique. Les études familiales n'ont pas encore permis d'élucider clairement le type de transmission héréditaire de la susceptibilité à l'asthme, à l'hyperréactivité bronchique et à l'allergie. La recherche d'association à des marqueurs génétiques connus, comme le complexe majeur d'histocompatibilité, ou à des polymorphismes anonymes de l'ADN, a déjà permis d'identifier des associations entre la sensibilité à certains allergènes et des gènes HLA particuliers et de suggérer une liaison génétique entre l'allergie et un gène situé sur le bras court du chromosome 11.

Marie-Hélène Dizier Alain Lockhart

\section{ADRESSES}

M.-H. Dizier : chargée de recherche (classe 2) da l'Inserm. Inserm U. 155, laboratoire d'anthropologie, 2, place Jussieu, 75006 Paris, France.

A. Lockhart : professeur des universités, praticien hospitalier. Service d'explorations fonctionnelles respiratoires, hôpital Cochin, 27 rue du Faubourg Saint-Jacques, 75014 Paris, France.

$m / s n^{\circ} 10$, vol. 7, décembre 91 e caractère familial de l'asthme est connu depuis très longtemps, cependant la composante génétique intervenant dans cette maladie n'est toujours pas clairement identifiée. En effet, l'étude génétique de cette affection est difficile en raison de la complexité de la maladie elle-même. Le caractère familial de la maladie peut s'expliquer par la composante génétique autant que par l'effet d'un environnement commun à des sujets ayant un même habitat, et il est difficile de préciser la part respective de ces deux facteurs De plus, l'asthme représente un phénotype complexe en raison de son association étroite avec l'hyperréactivité bronchique et l'allergie, pour lesquelles une composante génétique a pu être également montrée. Il existe vraisemblablement à la fois des facteurs génétiques spécifiques à chacune de ces trois affections, l'asthme, l'hyper-réactivité bronchique et l'allergie et des facteurs communs.

\section{Définition des phénotypes de l'asthme, de l'hyperréactivité bronchique et de l'allergie}

- L'asthme est une maladie identifiée depuis fort longtemps, mais sa définition est encore actuellement imprécise. En 1975, des experts de l'OMS en ont donné la définition suivante: "L'asthme est une affection chronique caractérisée par un bronchospasme récidivant résultant de la tendance à répondre par la dimi- 


\section{RÉFÉRENCES}

1. World Health Organization. Epidemio logy of chronic non-specific respiratory diseases. Bull WHO 1975 ; 52 : 251-9.

2. Bignon J, Brochard P, Voisin J. Maladies respiratoires spécifiques communes à plusieurs facteurs professionnels. Rev $\mathrm{Ma}$ Respir 1990 ; 7 (suppl. 2) : 107-18.

3. Woolcock AJ, Permutt S. Bronchial hyperresponsiveness. In : Handbook of Physiology. The Respiratory System, Vol III, Mechanic of Breathing Part 2. Bethesda, Maryland : American Physiological Society, 1986 : 727-36.

4. Cockroft DW, Hargreave FE. Airway hyperresponsiveness. Relevance of random population data to clinical usefulness. $A m$ Rev Respir Dis 1990; 142 : 497-500.

5. Pattemore PK, Asher MI, Harrison AC, Mitchell EA, Rea HM, Stewart AW. The interrelationship among bronchial hyperresponsiveness, the diagnosis of asthma, and asthma symptoms. Am Rev Respir Dis 1990 ; 142 : 549-54

6. Marsh DG. Historical introduction. In : March DG, Blumenthal MN, eds. Genetic and Environmental Factors in Clinical Allergy. Minneapolis : University of Minnesota Press, 1990 : 3-9.

7. Morton NE, Maclean CJ. Analysis of family resemblance. III. Complex segregation analysis of quantative traits. $A m J$ Hum Genet 1974 ; 26 : 489-503.

8. Bonney GE. On the statistical determination of major gene mechanisms in continuous human traits : regressive models. $A m$ $J$ Med Genet 1984; 18 : 731-49.

9. Morton NE. The detection and estimation of linkage between the genes for elliptocytosis and the $\mathrm{Rh}$ blood type. Am J Hum Genet 1956 ; 8 : 80-96.

10. Thomson G, Boldmer WF. The genetic analysis of HLA and disease associations. In : Dausset J, Svejgaard A, eds. HLA and Disease. Copenhague : Munksgaard, 1977 ; $8: 84-93$

11. Schwartz M. Heredity in bronchial asthma. Acta Allergol 1952 ; 5 (suppl. 11) : 212-8.

12. Edfors-Lubs ML. Allergy in 7000 twin pairs. Acta Allergol 1971; 26 : 249-85.

13. Snyder R, Collipp P, Green J. Growth and ultimate height of children with asthma. Clin Pediatr 1967 ; 6 : 389-92.

14. Leigh D, Marley E. Bronchial asthma. A genetic, population and psychiatric study. Oxford : Pergamon Press, 1967.

15. Sibbald B, Horn MEC, Brain EA, Gregg I. Genetic factors in childhood asthma. Thorax $1980 ; 35$ : 671-4. nution de calibre des voies aériennes à des stimuli d'intensité ou de niveau n'entraînant pas une telle diminution chez des sujets normaux "[1]. Plus récemment, en 1990, un groupe d'experts de la société de pneumologie de langue française définit l'asthme comme "une dyspnée paroxystique, survenant souvent la nuit, réversible soit spontanément ou sous l'effet des traitements et causée par une obstruction bronchique secondaire à des mécanismes immunologiques ou autres. Une caractéristique du syndrome est l'hyperréactivité bronchique à une variété d'agoniste " [2].

Dans cette nouvelle définition, le remplacement des mots affection ou maladie par syndrome et l'affirmation que l'asthme correspond à différents mécanismes traduisent les incertitudes sur la physiopathologie de l'asthme. En outre, l'emploi du mot obstruction bronchique, plutôt que bronchospasme, s'explique par l'intervention d'autres facteurs que la contraction du muscle lisse des voies aériennes, par exemple l'œdème inflammatoire et l'épaississement de la sousmuqueuse [3].

- L'hyperréactivité bronchique non allergénique, HRBNA, correspond à une obstruction bronchique démesurée en réponse à des agents physiques, chimiques ou pharmacologiques. L'hyperréactivité bronchique est un phénotype complexe à étudier. Premièrement, chez les asthmatiques, elle est d'autant plus accentuée que les symptômes sont plus sévères, mais peut manquer, à distance des crises, dans certains asthmes intermittents [4]. Deuxièmement, il n'y a pas de corrélation étroite entre la réponse bronchique aux stimuli agissant directement sur les effecteurs, histamine et acétylcholine par exemple, et à ceux dont le mode d'action est indirect et d'ailleurs mal connu, comme l'exercice physique. Les résultats d'une étude génétique pourraient donc être différents selon le stimulus utilisé. Troisièmement, l'hyperréactivité bronchique n'est pas spécifique de l'asthme et existe dans d'autres affections comme, par exemple, la bronchite chronique obstructive et la rhinite allergique. Enfin, des études transversales ont montré qu'il existait également des individus sains hyperréactifs [5].

- L'allergie était initialement définie comme la présence de différentes affections cliniques, volontiers, mais pas toujours, associées entre elles (l'asthme, la rhinite, l'eczéma...) et de réponses cutanées positives aux pneumo-allergènes courants. Une étape essentielle a été la découverte de l'immunoglobuline $\mathrm{E}$ ( $\mathrm{IgE}$ ) et, très vite, de l'association étroite entre l'allergie et l'IgE, suivie de la mise en évidence d'IgE spécifique d'un antigène donné chez des individus sensibilisés à cet antigène [6]. Toutefois la mise en évidence du phénotype clinique de l'allergie se heurte au fait que l'intensité et le nombre des réponses cutanées aux pneumoallergènes courants, ainsi que la concentration sérique en $\operatorname{IgE}$, décroissent avec l'âge. C'est une des raisons pour lesquelles la séparation en asthme allergique et asthme non allergique est actuellement controversée.

\section{Stratégies de détection de facteurs génétiques}

La mise en évidence de facteurs génétiques impliqués dans une maladie peut se faire par deux types d'approche.

\section{- Étude de prévalence familiale} La comparaison de la prévalence d'une maladie chez les apparentés d'individus atteints et chez les apparentés d'individus non atteints permet de montrer s'il existe une concentration familiale de la maladie. Une telle concentration familiale suggère l'effet de facteurs génétiques, mais, comme nous l'avons vu, elle peut également s'expliquer par l'effet d'un environnement familial partagé.

\section{- Étude de couples de jumeaux}

La comparaison du taux de concordance pour la maladie entre des couples de jumeaux monozygotes et des couples de jumeaux dizygotes est très informative pour rechercher l'effet d'une composante génétique dans la famille. En effet, les couples de jumeaux monozygotes sont génétiquement identiques tandis que des couples de jumeaux dizygotes se ressemblent génétiquement comme des frè- 
res ou sœurs, c'est-à-dire qu'il y a en moyenne $25 \%$ de ressemblance pour le génome entier. On peut supposer que la ressemblance pour l'exposition à l'environnement est la même entre les jumeaux monozygotes et entre les jumeaux dizygotes. Un taux de concordance pour la maladie plus élevé chez les couples de jumeaux monozygotes que chez les couples de jumeaux dizygotes permet donc de conclure à l'effet de facteurs génétiques. Par ailleurs, si le trait étudié ne dépend que de facteurs génétiques, on attend un taux de concordance de $100 \%$ chez les jumeaux monozygotes. Un taux de concordance inférieur à $100 \%$ permet alors de conclure à l'effet de facteurs environnementaux surajoutés.

- Analyse de ségrégation familiale Si l'effet de facteurs génétiques dans la susceptibilité à une maladie a été suggéré ou montré par l'une des approches précédentes, des études d'analyse de ségrégation familiale permettent ensuite de préciser le déterminisme génétique de la maladie. Une telle analyse [7] permet habituellement d'identifier le modèle génétique le plus simple expliquant le mieux la répartition du trait observée dans un échantillon de familles. Cette méthode permet en particulier de rechercher l'effet d'un gène majeur et/ou d'une composante polygénique (effet individuel minime de plusieurs gènes) et d'estimer la fréquence du gène majeur et son mode de transmission, récessif ou dominant.

Cependant, pour l'étude des maladies à étiologie complexe, dépendantes à la fois de facteurs génétiques et environnementaux, il est nécessaire de tenir compte de cet effet de l'environnement pour bien préciser la part de la ressemblance familiale expliquée par des facteurs génétiques et celle expliquée par l'habitat et le mode de vie familiaux communs. De plus, une différence dans l'exposition à des facteurs du milieu pouvant modifier l'effet de facteurs génétiques, des biais importants peuvent intervenir dans l'étude de la composante héréditaire si l'on ne prend pas en compte l'exposition aux facteurs environnementaux. Une nouvelle approche [8] a été développée récemment pour mieux répondre à ces problè$\mathrm{m} / \mathrm{s} n^{\circ} 10$, vol. 7 , décembre 91 mes. Elle permet d'étudier la ségrégation familiale d'un trait en tenant compte des expositions à différents facteurs de l'environnement. Il est alors possible d'étudier simultanément l'effet de déterminants génétiques (gène majeur et composante polygénique) et de facteurs environnementaux, et de rechercher une interaction entre ces deux composantes.

\section{- Utilisation des marqueurs généti- ques}

Il existe deux types d'étude utilisant l'information sur des marqueurs génétiques : l'analyse familiale, permettant de rechercher une liaison génétique entre le gène de la maladie et le marqueur, et l'étude " castémoin " pour rechercher une association.

- Recherche de liaisons génétiques par analyse familiale

Si l'effet d'un gène majeur est suggéré ou montré dans la susceptibilité d'une maladie, on peut, par des analyses statistiques, la méthode du lod score [9] ou la méthode des germains [10], rechercher une liaison génétique entre le gène de la maladie et un marqueur génétique. Pour cela, on étudie la ségrégation conjointe de la maladie et du marqueur dans des familles et, si cette ségrégation n'est pas indépendante, on conclut à l'effet d'un gène lié à ce marqueur. La mise en évidence d'une liaison génétique avec un marqueur permet ainsi de préciser la localisation chromosomique du gène de la maladie.

Les marqueurs étudiés peuvent être des systèmes génétiques connus, comme les systèmes HLA, $\mathrm{ABO}$, Rhésus..., ou des séquences d'ADN polymorphes, que les progrès de la génétique moléculaire permettent maintenant d'identifier.

- Recherche d'associations par étude "cas-témoins"

La comparaison des fréquences alléliques d'un marqueur entre des groupes de cas et de témoins permet également de rechercher une association entre le marqueur et la maladie. Cependant, une telle association suggère, mais ne prouve pas, l'effet d'un gène lié au marqueur étudié. Pour cela, il est nécessaire de faire une analyse de liaison sur des données familiales. L'intérêt actuel de ce type d'étude est souvent, en utilisant comme marqueurs des gènes " candidats " susceptibles d'intervenir euxmêmes dans la maladie, de préciser les allèles du marqueur associés à la maladie.

\section{Études familiales de l'asthme, de l'hyperréactivité bronchique et de l'allergie}

\section{- L'asthme}

Les études de prévalence familiale ont permis de montrer une concentration familiale de l'asthme, avec une prévalence de $13 \%$ chez les apparentés d'asthmatiques et une prévalence de $4 \%$ chez les apparentés de nonasthmatiques [11]. Une étude portant sur 7000 couples de jumeaux [12] a permis de confirmer l'effet d'une composante génétique dans l'asthme, le taux de concordance chez les jumeaux monozygotes étant de $19 \%$ et chez les jumeaux dizygotes de $5 \%$. Cependant, le taux de concordance peu élevé chez les jumeaux monozygotes montre un effet important d'une composante environnementale. Les études d'analyse de ségrégation familiale ont donné des résultats très discordants $[13,14]$ et le déterminisme génétique de l'asthme n'est toujours pas clairement défini, vraisemblablement en raison de sa complexité. De plus, une étude distinguant l'asthme allergique de l'asthme non allergique est en faveur de l'effet de facteurs génétiques indépendants dans l'asthme et dans l'allergie [15].

\section{- L'hyperréactivité bronchique}

La corrélation intrapaire pour la réactivité bronchique est plus élevée chez les couples de jumeaux monozygotes $(67 \%)$ que chez les jumeaux dizygotes $(34 \%)[16]$. Différentes études ont montré que la distribution de la réactivité bronchique correspond à un mélange de deux populations distinctes, ce qui peut suggérer l'effet d'un gène majeur $[17,18]$. Cependant, l'étude de la ségrégation familiale de la réactivité bronchique [19] a confirmé l'hypothèse d'une composante génétique mais non l'effet d'un gène majeur; le mode de transmis- 


\section{RÉFÉRENCES}

16. Hopp RJ, Bewtra AK, Watt GD, Nair NM, Townley RG. Genetic analysis of allergic disease in twins. J Allergy Clin Immunol $1984 ; 73$ : 265-70.

17. Longo G, Strinati R, Poli F, Fumi F. Genetic factors in non specific bronchial hyperreactivity. An epidemiologic study. $A m$ $J$ Dis Child 1987 ; 141: 331-4.

18. Hopp RJ, Bewtra MD, Biven R, Nair NM, Townley RG. Bronchial reactivity pattern in non asthmatic parents of asthmatics. Ann Allergy 1988 ; 61 : 184-6.

19. Townley RG, Bewtra A, Wilson AF, et al. Segregation analysis of bronchial response to methacholine inhalation challenge in families with and without asthma. J Allergy Clin Immunol $1986 ; 77$ : 101-7.

20. Pauwels R, Van der Straeten M, Weyne J, Bazin H. Genetic factors in nonspecific bronchial reactivity in rats. Eur $J$ Respir Dis 1985 ; 66 : 98-104

21. Levitt RC, Mitzner W. Expression of airway hyperreactivity or acetylcholine as a simple autosomal recessive trait in mice. FASEB J 1988 ; 2 : 2605-8.

22. Levitt RC, Mitzner W. Autosomal recessive inheritance of airway reactivity to 5-hydroxytryptamine. J Appl Physiol 1989 ; 67 : 1125-32.

23. Wang CG, Dimaria G, Bates JHT, Guttmann RD, Martin JG. Methacholineinduced airway reactivity of inbred rats. $J$ Appl Physiol 1986; 61: 2180-5.

24. McDonald DM. Respiratory tract infection increase susceptibility to neurogenic inflammation in the rat trachea. Am Rev Respir Dis 1988 ; 137 : 1432-40.

25. Lubs ML. Empiric risks for genetic counseling in familics with allergy. J Pediatr $1972 ; 80: 26$.

26. Marsh DG, Bias WB, Ishizaka K Genetic control of basal serum immunoglobulin $\mathrm{E}$ level and its effect on specific reaginic sensitivity. Proc Natl Acad Sci 1974 71 : 3588-92.

27. Gerrard JW, Rao DC, Morton NE. A genetic study of immunoglobulin E. Am J Hum Genet 1978 ; 30 : 46-58.

28. Meyers DA, Beaty TH, Freidhoff LR, Marsh DG. Inheritance of total serum IgE (basal levels) in man. Am J Hum Genet $1987 ; 41: 51-62$.

29. Watanabe N, Kojima S, Ovary Z. Suppression of $\mathrm{IgE}$ antibody production in $\mathrm{SJL}$ mice. Non specific suppressor T cells. J Exp 1044 sion de la réactivité bronchique doit donc être plus complexe et dépend vraisemblablement de l'interaction entre des facteurs génétiques et environnementaux. L'existence de facteurs génétiques dans la susceptibilité à l'HRBNA (hyperréactivité bronchique non allergénique) paraît bien établie dans différentes espèces ou races d'animaux. Par sélection, il a été possible d'obtenir des races de cobayes, de rats et de souris ayan une HRBNA à- divers agents pharmacologiques. Chez le rat et la souris, l'HRBNA peut être sélective visà-vis de certains agonistes, par exemple la métacholine, la sérotine ou les tachykinines et les transmissions de ces HRBNA sélectives seraient monogéniques et autosomiques [20-22] Cependant, la variabilité entre rats de même race pourrait être au moins aussi, si ce n'est plus importante que la variabilité entre races [23], ce qui fait penser que l'environnement a un rôle très important. D'ailleurs, il est maintenant bien établi que les infections banales des voies aériennes qui ne sont pas contrôlées dans la plupart des expériences précitées sont capables de modifier la structure des voies aériennes et la réponse des bronches à des stimuli pharmacologiques ultérieurs [24].

\section{- L'allergie}

Les études portant sur les différentes manifestations cliniques de l'allergie : asthme, rhinite, eczéma... (Tableau I), ont montré une concentration familiale [25] et l'étude de couples de jumeaux a permis de confirmer une composante génétique [12]. Pour le taux d'IgE, des études sur les couples de jumeaux ont montré une corrélation de $82 \%$ chez les jumeaux monozygotes et de $46 \%$ chez les jumeaux dizygotes [16]. L'allergie étant une maladie complexe et très hétérogène cliniquement, les analyses de ségrégation familiale ont plus particulièrement porté sur la concentration totale d'IgE dans le sérum comme marqueur biologique de l'allergie. Différentes études ont montré, outre l'effet d'une composante polygénique, l'effet d'un gène majeur récessif [26-28]. Ce résultat est compatible avec ceux obtenus chez la souris, montrant une transmission monogénique récessive pour un taux
d'IgE élevé [29]. Cependant, chez l'homme, d'autres études ont donné des résultats très différents $[30,31]$, l'une d'entre elles suggérant une hétérogénéité génétique (c'est-à-dire l'effet de différents gènes) dans la régulation du taux d'IgE [32]. La discordance des résultats pourrait s'expliquer par des problèmes liés au mode de sélection des familles et également par la variation saisonnière du taux d'IgE, la stratégie de recueil des données n'étant pas homogène dans les différentes études [28].

\section{Études utilisant des marqueurs génétiques}

Différentes études ont été faites pour rechercher soit, par des études familiales, une liaison génétique de l'asthme et de l'allergie avec des marqueurs génétiques, soit une association par des études cas-témoins. Le système HLA était un marqueur potentiellement intéressant pour l'étude de ces maladies. En particulier, la découverte du contrôle de la réponse immune spécifique chez la souris par les gènes Ir, liés au système majeur d'histocompatibilité chez cet animal [33], a été le point de départ des recherches d'association des gènes du système HLA avec les réponses immunes spécifiques chez l'homme.

D'autres marqueurs ont été étudiés par la suite, en particulier des polymorphismes situés sur le bras court du chromosome 11 .

\section{- L'asthme}

La recherche d'association avec le système HLA par étude cas-témoins a donné des résultats très discordants. Si une augmentation des antigènes HLA A1 et B8 a pu être observée chez des sujets asthmatiques [34, 35], les résultats obtenus dans d'autres études ont souvent été très différents [36, 37]. Une association a été récemment mise en évidence entre l'asthme à l'aspirine et l'antigène HLA DQw2 [38], et les recherches se poursuivent actuellement dans ce sens pour rechercher des associations avec d'autres asthmes spécifiques.

Deux études familiales ont montré que l'asthme et les antigènes du système HLA ne se transmettaient pas indépendamment chez des cou- 


\begin{tabular}{|c|c|r|r|}
\hline \multicolumn{3}{|c|}{ Tableau I } \\
\multicolumn{4}{|c|}{$\begin{array}{c}\text { PRÉVALENCE D'ALLERGIE CHEZ LES APPARENTÉS } \\
\text { DU 1er DEGRÉ D'INDIVIDUS ALLERGIQUES }\end{array}$} \\
\hline \multirow{2}{*}{ Index } & Allergie & \multicolumn{2}{c|}{ Prévalence (\%) } \\
\cline { 3 - 4 } & & Population témoin & Apparentés du 1er degré \\
& & 3,8 & 9,2 \\
Asthme & Asthme & 14,8 & 25,2 \\
& Rhinite & 2,5 & 4,3 \\
& Eczéma & 3,8 & 6,0 \\
& Asthme & 14,8 & 24,1 \\
& Rhinite & 2,5 & 3,3 \\
& Eczéma & 3,8 & 6,2 \\
& Asthme & 14,8 & 24,1 \\
Eczéma & Rhinite & 2,5 & 7,7 \\
& Eczéma & & \\
\hline
\end{tabular}

D’après [25].

ples de frères et sœurs $[39,40]$. Cette liaison génétique semble spécifique de l'asthme allergique et est en faveur de l'effet d'un gène situé dans la région du système HLA et impliqué dans cette forme de la maladie.

\section{- L'allergie}

\section{- Réponse des IgE spécifiques}

La recherche d'association entre la sensibilisation à un antigène spécifique et une spécificité HLA a permis de montrer des associations à des allergènes purifiés, de petite taille plutôt qu'à des molécules de très grande taille susceptibles de porter de multiples épitopes [41]. C'est ainsi qu'a été mise en évidence l'association entre la sensibilisation spontanée à un antigène d'Ambrosia artemisiifolia (Amb a V) et les antigènes DR2/Dw2 (Tableau 2) [42]. Les sujets Dw2 + mais sans immunoglobulines spécifiques contre $A m b$ a $V$ sont capables de faire de tels anticorps en cas d'immunisation par des injections répétées alors que les sujets Dw2 - ne le sont pas [43, 44]. Cette expérience confirme le rôle de l'environnement en démontrant que la quantité totale d'allergènes à laquelle l'individu a été exposée est importante pour que s'extériorise le phénotype clinique. De même, la liaison entre l'antigène DR3 et la sensibilisation aux allergènes de Lolium perennae (Lol p1, Lol p2 et Lol p3) [44] et de dermatophagoïdes [45] a été mise en évidence. Inversement, la faible réponse à un antigène du cèdre japonais $\left(C_{\tau y} j 1\right.$ ) est associée à certains antigènes HLA-DQ, ce qui est un argument en faveur du rôle protecteur de gènes DQ [45, 46].

Grâce aux récents progrès de la géné-

Tableau II

ASSOCIATION ENTRE DES ANTIGĖNES HLA ET LA RÉPONSE IgE SPÉCIFIQUE DES ALLERGĖNES DE POLLEN [43]

\begin{tabular}{|l|c|c|}
\hline \multicolumn{1}{|c|}{ Allergène } & Association HLA & $p^{*}$ \\
\hline Ambrosia artemisiifolia & DR2/DW2 & $<10^{-9}$ \\
Amb a V & & $<10^{-3}$ \\
Lolium perenne & DR3 & $10-3$ \\
Lol p I & DR3 & $<10^{-5}$ \\
Lol p II & DR3 & \\
Lol p III & DR \\
\hline
\end{tabular}

* p estimé en utilisant le test exact de Fisher.

D'après [44].

$\mathrm{m} / \mathrm{s} n^{\circ} 10$, vol. 7, décembre 91

tique moléculaire, les recherches se poursuivent afin de préciser les spécificités de la région HLA-DR associées à la réponse à des allergènes spécifiques, en étudiant les régions hypervariables des gènes $\mathrm{DR} \beta, \mathrm{DQ} \alpha$ et $\mathrm{DQ} \beta$ [43]. Des premiers résultats ont été obtenus dans la sensibilisation à $A m b a \cdot V$ [43]. Les résultats de notre étude sur la sensibilisation à l'aspirine ont montré que la combinaison des allèles $\mathrm{DQ} \beta$ 2-3, 1 et $\mathrm{DP} \beta$ 4,2-x étäit associée à un risque relatif de 54 pour la sensibilité à l'aspirine chez des sujets allergiques [47]. Là structure des épitopes responsables de la sensibilisation spécifique commence à être élucidée, ce qui pourrait permettre d'expliquer certaines sensibilisations croisées apparemment paradoxales et des sensibilisations spécifiques différentes chez des sujets ayant la' même spécificité HLA de classe II. Par exemple, certaines séquences peptidiques analogues ont été mises en évidence dans l'agglutinine du germe de blé et l'allergène d'Ambrosia artemisiifolia Amb a $V$ [44]. En outre, il y a un relatif manque de spécificité de la présentation de peptides par les molécules HLA de classe II, les mêmes molécules pouvant présenter des peptides différents [48, 49].

\section{- Réponse des IgE non spécifiques}

Aucune liaison génétique n'a pu être mise en évidence entre le taux d'IgE total et le système HLA ou d'autres marqueurs génétiques (systèmes $\mathrm{ABO}$, Rhésus...) $[26,50]$. En revanche, une étude portant sur un échantillon de familles allergiques a montré une liaison génétique entre l'atopie et un polymorphisme génétique situé sur le bras court du chromosome 11 [51]. Dans cette étude, l'atopie était définie par la présence d'au moins un des critères suivants : un taux d'IgE spécifique élevé pour au moins un allergène, une réponse positive à un test cutané pour au moins un allergène et un taux d'IgE total élevé. Cette étude suggère donc l'effet d'un gène situé sur le chromosome 11 dans l'atopie. Ce résultat est intéressant en raison de la présence probable sur le chromosome 11 de nombreux gènes codant pour des molécules de surface des cellules $T$, celles-ci ayant un rôle important dans la réponse des $\operatorname{IgE}$. Cette liaison géné- 
30. Meycrs DA, Bias WB, Marsh DG. A genetic study of total IgE in the Amish. Hum Hered $1982 ; 32$ : 15-23.

31. Hasstedt SJ, Meyers DA, Marsh DG. Inheritance of immunoglobulin $\mathrm{E}$ : genctic model fitting. Am J Hum Genet 1983; 14 : 61-6.

32. Blumenthal MN, Namboodiri KK, Mendell N, Glcich G, Elston RC, Yunis E. Genetic transmission of serum IgE levels. Am J Med Genet 1981 ; 10 : 219-28.

33. McDevitt HO, Tyan ML. Genctic control of the antibody response in inbred mice : transfer of response by splcen cells and linkage to the major histocompatibility (H-2) locus. J Exp Med $1968 ; 128$ : 1-11.

34. Thorsby E, Engset A, Lie SO. HLA antigens and susceptibility to diseases. A study of patients with acute lymphoblastic leukemia, Hodgkins's disease and childhood asthma. Tissue Antigens 1971 ; 1 : 147-52.

35. Soothill JF, Stokes CR, Turner MW, Norman AP, Taylor B. Predisposing factors and the development of reaginic allergy in infancy. Clin Allergy $1976 ; 6$ : 305.

36. Turton CMG, Morris L, Buckingham JA, et al. Histocompatibility antigens in asthma : population and family studies. Thorax 1979 ; 34 : 670-6.

37. Morris MJ, Faux JA, Ting A, Morris PJ, Land DJ. HLA-A, B and C and DR antigens in intrinsic and allergic asthma. Clin Allergy $1980 ; 10: 173-9$.

38. Mullarkey MF, Thomas PS, Hansen JA, Webb DR, Nisperos B. Association of aspirin-sensitive asthma with HLADQw2. Am Rev Respir Dis 1986; 133 : 261-3.

39. Hafez M, Zedan M, El-Shennawy FA, Abd El-Hafez SA, El-Khayat H. HLA Antigens and extrinsic bronchial asthma. $J$ Asthma 1984 ; 21 : 259-63.

40. Caraballo LR, Hernandez M. HLA haplotype segregation in families with allergic asthma. Tissue Antigens $1990 ; 35$ : 182-6.

41. Marsh DG. Immunogenetic and immunochemical factors determining immune responsiveness to allergens : studies in unrelated subjects. In : Marsh DG, Blumenthal MN, eds. Genetic and Environmental Factors in Clinical Allergy. Minneapolis : University of Minnesota Press, 1990 : 97-123.

42. March DG, Meyers DA, Freidhoff LR, et al. HLA-Dw2 : a genetic marker for human immune response to short ragweed pollen allergen Ra5.II. Response after ragweed immunotherapy. J Exp Med 1982;
43. Marsh DG. Molecular studies of human immune recognition of allergens. Allergy $1988 ; 43: 7-9$

44. Marsh DG, Zwollo P, Ansari AA Toward a total human immune response finger print : the allergy model. In Said EL, Shami A, Merrett TG, eds. Allergy and Molecular Biology. Oxford/New York: Pergamon Press, 1989: 65-82.

45. O'Hehir RE, Mach B, Bcrte C, et al. Direct evidence for a functional role of HLA-DR $\beta 3$ gene products in the recognition of Dermatophagoides spp by helper T celle clones. J Immunol 1990 ; 62 : 635-40.

46. Sasazuki T, Nishimura $Y$, Moto $M$ Oht a N. HLA-linked genes controlling immune response and disease susceptibility Immunol Rev 1983 ; 70 : 52-75.

47. Périchon B, Gu XF, Ostinelli J, et al. Intriguing prevalence of coeliac disease associated HLA-class II DNA haplotype in aspirin sensitization of atopic asthmatic patients. American Academy of Allergy and Immunology 2-6 March. San Francisco. Oxford : Blackwel Scientific Publication Ltd 1991 (sous pressc).

48. Settc A, Buus S, Appela E, Adorini L Grey HM. Structural requirements for the interaction between class II MHC molecules and peptide antigens. Immunol Res 1990 $9: 2-7$.

49. Sette A, Buus S, Colon S, Miles C, Grey HM. Structural analysis of peptides capable of binding to more than 1 antigen. $J$ Immunol 1989 ; 142 : 35-40

50. Meyers DA, Hasstedt SJ, Marsh DG The inheritance of immunoglobulin E : linkage analysis. Am J Med Genet $1983 ; 16$ : $575-82$

51. Cookson WOCM, Faux JA, Sharp PA, Hopkin JM. Linkage between immunoglobulin $\mathrm{E}$ responses underlying asthma and rhinitis and chromosome 11q. Lancet 1989 1 : 1292-4.

52. Bcasley R, Roche WR, Roberts JA, Holgatc ST. Cellular cvents in the bronch in mild asthma and after bronchial provocation. Am Rev Respir Dis 1989; 139 806-17

53. Brewster CEP, Howarth PH, Djukano vic $R$, Wilson J, Holgate ST, Roche WR. Myofibroblasts and subepithelial fibrosis in bronchial asthma. Am J Respir Cell Mol Biol $1990 ; 3: 507-11$.

54. Rossi GA, Sacco O, Balbi B, et al. Human ciliated bronchial epithelial cells expression of the HLA-DR antigens and of the HLA-DR alpha gene, modulation of the HLA-DR antigens by gamma-interferon and antigen-presenting function in the mixed leukocyte reaction. Am J Respir Cell Mol Biol $1990 ; 3: 431-40$. tique demande néanmoins à être confirmée en reproduisant cette étude sur d'autres échantillons de familles.

Il y a donc probablement l'effet d'au moins deux facteurs génétiques indépendants dans le contrôle de la réponse des $\operatorname{IgE}$, l'un lié au système HLA pour la réponse immune spécifique des $\operatorname{IgE}$ et l'autre non lié au système HLA dans la régulation de la production totale des IgE. De la même manière, il serait intéressant de préciser l'implication exacte du gène localisé sur le chromosome 11, d'une part, dans la réponse des $\mathrm{IgE}$ spécifiques et, d'autre part, dans la régulation des IgE totales.

\section{Conclusion et perspectives}

Actuellement, il existe une grande incertitude sur la composante génétique de l'asthme. Une seule certitude est que le déterminisme génétique de l'asthme ne correspond pas à un modèle de transmission simple, monogénique. Pour progresser dans l'étude de cette maladie, il faudrait pouvoir évaluer les parts respectives des facteurs génétiques et des facteurs environnementaux. Pour cela, il est nécessaire de tenir compte dans l'analyse de ségrégation familiale, de facteurs de milieu, comme le tabagisme, les infections virales... Il serait également important de préciser les effets de facteurs génétiques communs ou indépendants dans l'asthme, l'hyperréactivité bronchique et l'allergie.

Si la recherche sur l'asthme va certainement bénéficier rapidement des progrès de la biologie moléculaire, il est certain que, dans l'état actuel des connaissances, les études en cours portent plus sur les facteurs génétiques de l'allergie que sur ceux de l'asthme proprement dit. Plusieurs voies de recherche sont d'ores et déjà ouvertes. Trois d'entre elles concernent l'allergie. La première est de confirmer l'existence d'une liaișon génétique entre l'allergie et un polymorphisme situé sur le bras court du chromosome 11, de trouver des marqueurs de plus en plus proches du gène, de cloner ce gène, d'identifier la protéine et d'en comprendre le mode d'action. La deuxième est de préciser les mécanismes moléculaires de l'allergie en étudiant le rôle des molécules HLA de 
classe II dans cette réaction. La troisième est d'étudier des gènes candidats, n'appartenant pas au système HLA, tels que ceux supposés intervenir dans le contrôle de la synthèse des $\mathrm{IgE}$, par exemple les gènes codant pour l'interleukine 4 et l'interféron gamma et ceux codant pour les récepteurs des cellules $\mathrm{T}$ ou certaines molécules d'adhérence comme ICAM 1. La quatrième est de chercher des marqueurs génétiques liés à l'hyperréactivité bronchique même et non plus à l'allergie : la mise en évidence chez la souris de loci chromosomiques associés à l'hyperréactivité bronchique non allergénique pour un agent pharmacologique pourrait faciliter le repérage de loci homologues dans le génome humain [22]. En outre, l'introduction en pratique courante de la fibroscopie bronchique rend possible l'identification de médiateurs et de populations cellulaires impliqués dans l'asthme [52-54], et on peut espérer que cela permettra de disposer de gènes candidats à étudier, autres que ceux en jeu dans la réponse des IgE. Une autre alternative est de poursuivre la recherche d'association avec de nouveaux polymorphismes génétiques, comme cela a été fait pour le chromosome 11.

Une vaste enquête multicentrique commanditée par l'Inserm et coordonnée par F. Kauffman (U169 Inserm) est en train de commencer en France. Il est prévu de sélectionner un échantillon de 400 familles d'asthmatiques et de recueillir, pour tous les sujets concernés, les données suivantes : le phénotype clinique de l'asthme, de la réactivité bronchique et de l'allergie, l'exposition aux facteurs de milieu - tabagisme, conditions de logement, exposition professionnelle... L'objectif de cette étude est d'étudier simultanément les facteurs génétiques et environnementaux impliqués dans l'asthme, l'hyperréactivité bronchique et l'allergie, et de préciser les facteurs génétiques communs et indépendants dans ces trois phénotypes. Parallèlement, la recherche de marqueurs génétiques liés aux phénotypes étudiés sera entreprise. On peut espérer qu'à partir de cette étude il sera possible de préciser la composante génétique de l'asthme et que cela permettra ensuite de mieux en comprendre les mécanismes biologiques

$m / s n^{\circ} 10$, vol. 7 , décembre 91

\section{Summary}

Genetics of asthma

Asthma is a multifactorial disease, influenced by both genetic and environmental factors. This affection is strongly associated with bronchial hyperresponsiveness and allergy, which are also partly genetically determined. Familial studies of asthma, bronchial hyperresponsiveness and allergy have not suggested any simple mode of transmission, i.e. depending on the effect of a single gene. Moreover, there are probably both specific and common genetic factors, acting on such phenotypes. Nevertheless, studies with genetic markers of the HLA system (the major histocompatibility complex), lead to interesting results. Particularly, associations have been shown between HLA antigens and the specific allergic responses to many allergens. In asthmatic families, co-transmission of HLA and allergic asthma has been documented.

Advances in molecular genetics now allow study of new genetic polymorphisms. A recent study in allergic families has shown a linkage between allergy and a polymorphism located on the short arm of the chromosome 11, suggesting the effect of a gene of the chromosome 11 in allergy.
TIRÉS A PART

M. H. Dizier. 